\title{
Scaling laws for positron production in laser-electron-beam collisions
}

\author{
T. G. Blackburn,,${ }^{1, *}$ A. Ilderton, ${ }^{2}$ C. D. Murphy, ${ }^{3}$ and M. Marklund ${ }^{1}$ \\ ${ }^{1}$ Department of Physics, Chalmers University of Technology, SE-41296 Gothenburg, Sweden \\ ${ }^{2}$ Centre for Mathematical Sciences, Plymouth University, Devon PL4 8AA, United Kingdom \\ ${ }^{3}$ York Plasma Institute, Department of Physics, University of York, York YO10 5DD, United Kingdom
}

(Received 23 May 2017; published 24 August 2017)

\begin{abstract}
Showers of $\gamma$ rays and positrons are produced when a high-energy electron beam collides with a superintense laser pulse. We present scaling laws for the electron-beam energy loss, the $\gamma$-ray spectrum, and the positron yield and energy that are valid in the nonlinear, radiation-reaction-dominated regime. As an application we demonstrate that by employing the collision of a $>\mathrm{GeV}$ electron beam with a laser pulse of intensity $>5 \times 10^{21} \mathrm{~W} \mathrm{~cm}^{-2}$, today's high-intensity laser facilities are capable of producing $O\left(10^{4}\right)$ positrons per shot via light-by-light scattering.
\end{abstract}

DOI: 10.1103/PhysRevA.96.022128

\section{INTRODUCTION}

Electron-positron pair creation by the interaction of light with light is one of the simplest processes in quantum electrodynamics (QED). Thus far, however, the two-photon process has not been detected in experiment [1-3], and the observation of multiphoton pair creation could only be accomplished by colliding the high-energy electron beam of the SLAC facility and an intense laser pulse [4-6]. It is expected that the next generation of high-intensity lasers [7-10] will create electromagnetic fields of sufficient magnitude such that the nonlinear analog of the Breit-Wheeler process becomes dominant [11] (see also [12,13]). As such fields are only otherwise found in extreme astrophysical environments [14-17], the prospect of studying plasma dynamics under these conditions in the laboratory has attracted considerable interest [18-20].

High-power laser facilities are already used to generate positrons via the Bethe-Heitler process, which converts bremsstrahlung photons produced by the deceleration of electrons in high- $Z$ material. The energetic electrons themselves are generated via direct illumination of the solid target [21-23] or by laser-wake-field acceleration [24-26]. In the case that the target electromagnetic field should be provided purely by light, Bell and Kirk [27] proposed an advantageous setup of electrons accelerated by counterpropagating, circularly polarized lasers, which is anticipated to create critical-density pair plasmas for laser intensities $>7 \times 10^{23} \mathrm{~W} \mathrm{~cm}^{-2}$ [28-32]. High-energy positrons may also be generated by the irradiation of a solid [33] or near-critical target $[34,35]$ with a laser of similar intensity.

Even though the highest intensity reached by currently available laser systems $\left(1 \times 10^{22} \mathrm{~W} \mathrm{~cm}^{-2}\right.$ [36] ) does not reach this level, it is still possible to explore nonlinear Breit-Wheeler pair creation in these facilities by employing the head-on collision of a high-energy electron beam and an intense laser pulse. This is because, in the rest frame of the electrons, the laser electric field amplitude is boosted by a factor $\gamma \gg 1$. As the electron propagates through the laser pulse, it loses energy by the emission of photons, which themselves interact with the laser fields to produce pairs [37,38], as shown in Fig. 1. The experimental setup can be made entirely optical if the necessary ultrarelativistic electron beam is obtained by laser-wake-field acceleration [39-41]. Such a configuration has already been used to generate $\mathrm{MeV} \gamma$ rays via nonlinear Thomson scattering $[42,43]$ and has been studied as a probe of quantum radiation reaction [44-47].

Here we consider the collision of a $\mathrm{GeV}$ electron beam with a laser pulse of intensity $>1 \times 10^{21} \mathrm{~W} \mathrm{~cm}^{-2}$ and present a set of analytical scaling laws for the electron energy loss, the photon spectrum, and the number and energy of positrons produced. This investigation complements the existing literature as it bridges the gap between analytic calculations in QED [48-55] and the use of large-scale particle-in-cell (PIC) simulations [56,57] that include QED processes by Monte Carlo sampling of rates evaluated in the locally constant field approximation [58,59].

The paper is organized as follows. First we derive an approximation for the pair-creation probability of a single high-energy photon colliding with an intense laser pulse in Sec. II. Then we consider producing these $\gamma$ rays via the inverse Compton scattering of an electron beam. We show in Sec. III A that high-energy photon production is maximized in the leading edge of the pulse near a point we call the "effective center"; identifying this region lets us estimate the electron energy loss in Sec. III B and derive an expression for the photon spectrum that accounts for radiation reaction in Sec. III C. We present scaling laws for the number and mean energy of the positrons arising from pair creation of these photons in Secs. IV A and IV B, respectively. Finally, we show how the colliding beams' finite sizes and offset affect the positron yield. Natural units $\hbar=c=1$ are used throughout.

\section{PAIR-CREATION PROBABILITY FOR A SINGLE PHOTON}

The importance of QED effects when photons and electrons interact with a strong electromagnetic field is governed by the quantum nonlinearity parameter [60-62]

\footnotetext{
*tom.blackburn@ chalmers.se
}

$$
\chi=\frac{e \sqrt{-(F p)^{2}}}{m^{3}} .
$$




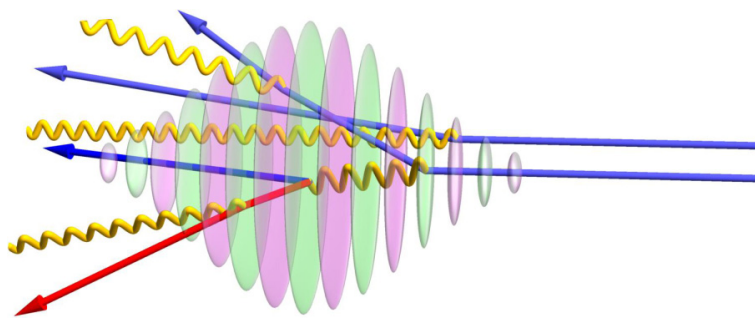

FIG. 1. Ultrarelativistic electrons (blue) collide with a counterpropagating laser pulse (magenta, green) and lose energy by emitting photons (yellow). Positrons (red) are created when photons undergo the nonlinear Breit-Wheeler process.

Here $e$ and $m$ are the electron charge and mass, $F$ is the electromagnetic field tensor, and $p$ the particle four-momentum. $(\chi$ is used to refer to electrons only; $\chi_{\gamma}$ and $\chi_{+}$are used for photons and positrons, respectively.) $\chi$ compares the magnitude of the electric field in the electron rest frame to that of the critical field of QED: $E_{\text {crit }}=m^{2} / e=1.326 \times 10^{18} \mathrm{~V} \mathrm{~m}^{-1}$ [63]. Even though the equivalent intensity, $3 \times 10^{29} \mathrm{~W} \mathrm{~cm}^{-2}$, is beyond our present capability, it is possible to reach $\chi \sim 1$ by colliding ultrarelativistic particles with weaker fields.

We consider a linearly polarized electromagnetic wave with Gaussian temporal profile as a simple model of a laser pulse, with amplitude $E=\left(m \omega_{0} a_{0} / e\right) \sin \phi \exp \left(-\ln (2) \phi^{2} /\left(2 \pi^{2} n^{2}\right)\right)$ at phase $\phi$. Here $a_{0}$ is the usual dimensionless strength parameter, $\omega_{0}=1.24 \mathrm{eV} /(\lambda / \mu \mathrm{m})$ is the wave frequency, and $n$ is the number of cycles corresponding to the pulse duration $\tau$, defined to be the full width at half maximum (FWHM) of the intensity profile. For now we consider only collisions with plane waves. We introduce a finite size for both the electron beam and the laser pulse to reach our final result in Sec. IV C.

In electromagnetic fields with $a_{0} \gg 1$, the formation length of QED processes is much smaller than the characteristic length scale of the external field, and we may use the locally constant field approximation (LCFA) [61]. This permits probabilities and rates to be calculated in an equivalent system of constant fields that have the same local value of $\chi$. It underlies numerical studies of highly intense laser-plasma interactions, where the electromagnetic fields have complex spatial and temporal structure that make direct analysis from strong-field QED unfeasible [58,59]. The field structure we consider here is much simpler, so the LCFA is key to keeping all our results analytical.

When a photon collides with an intense laser pulse, the dominant QED process is nonlinear Breit-Wheeler pair creation, which is first order in the fine-structure constant $\alpha$ but all orders in the coupling $a_{0}$ to the strong background field. The probability per unit phase of electron-positron pair creation for a photon with energy $\omega$ and nonlinearity parameter $\chi_{\gamma}$ is

$$
\frac{d P_{ \pm}}{d \phi}=\frac{W_{ \pm}}{2 \omega_{0}}=\frac{\alpha m^{2} \chi_{\gamma} T\left(\chi_{\gamma}\right)}{2 \omega_{0} \omega}
$$

where (see Appendix A for details) we follow Erber [60] and adopt the approximation $T\left(\chi_{\gamma}\right) \simeq \frac{0.16}{\chi_{\gamma}} K_{1 / 3}^{2}\left(\frac{4}{3 \chi_{\gamma}}\right)$, with $K_{\nu}(x)$ a modified Bessel function of the second kind. The probability in Eq. (2) is strongly suppressed for $\chi_{\gamma} \ll 1$.
We determine the probability that a photon pair-creates when colliding with an intense laser pulse, $P_{ \pm}$, in the following way. Starting from Eq. (2) we integrate over $\phi$ and use a saddle-point approximation to determine the contribution to the pair-creation probability at each local maximum, calling this $P_{i}$. Then given $P_{ \pm}=\sum_{i} P_{i}$ we replace the sum over $i$ with an integral and evaluate it using another saddle-point approximation.

Let $\phi_{i}$ be the phases at which the wave amplitude is (locally) maximized and $\chi_{i} \equiv \chi_{\gamma}\left(\phi_{i}\right)$ the associated photon nonlinearity parameter. Then the contribution to the probability from phases near $\phi_{i}$ is, using Eq. (2),

$$
P_{i}=\frac{0.16 \sqrt{3 \pi} \alpha m^{2}}{\sqrt{8} \omega_{0} \omega \sqrt{-\chi_{i}^{\prime \prime}}}\left[\frac{\chi_{i}^{2} K_{1 / 3}^{5}\left(\frac{4}{3 \chi_{i}}\right)}{K_{2 / 3}\left(\frac{4}{3 \chi_{i}}\right)+K_{4 / 3}\left(\frac{4}{3 \chi_{i}}\right)}\right]^{1 / 2},
$$

where $\chi_{i}^{\prime \prime} \equiv \chi_{\gamma}^{\prime \prime}\left(\phi_{i}\right)$. In a monochromatic plane wave, or a pulse with sufficiently slowly varying envelope, we have $\phi_{i}=\pi / 2+i \pi$ and $\chi_{i}^{\prime \prime}=-2 a_{0} \omega_{0} \omega / m^{2}$. Provided that $n$, the number of pulse cycles corresponding to FWHM duration, satisfies $n>2$, we can use these relations to determine $P_{i}$ as an analytical function of the index $i$. To evaluate the sum, we replace $\sum_{i} \rightarrow \int d i$ and perform the integration using the Laplace method, noting that the dominant contribution arises for $i=-1 / 2$, at the pulse center.

We find that the total probability for pair creation when a photon with energy $\omega$ collides with a linearly polarized laser pulse that has strength parameter $a_{0}$, frequency $\omega_{0}$, and (FWHM) number of cycles $n$ is

$$
P_{ \pm} \simeq \alpha a_{0} n \mathcal{R}\left(\frac{2 a_{0} \omega_{0} \omega}{m^{2}}\right),
$$

where we have introduced an auxiliary function $\mathcal{R}$ that is a function only of the photon nonlinearity parameter. $\mathcal{R}$ may be expressed analytically in terms of Airy functions, but as the underlying rate is being treated approximately, we introduce the following functional fit for compactness:

$$
\mathcal{R}(x)=\frac{0.453 K_{1 / 3}^{2}\left(\frac{4}{3 x}\right)}{1+0.145 x^{1 / 4} \ln (1+2.26 x)+0.330 x} .
$$

This fit is accurate to the analytical expression to within $1 \%$.

We compare the scaling law in Eq. (4) to the result of numerical integration of Eq. (2) in Fig. 2. Agreement is excellent across the full range of explored parameters: we capture the superexponential rise with increasing laser peak intensity and photon energy, that the pair yield scales linearly with pulse length, and that it does not scale with wavelength [provided that the wavelength is smaller than the pulse FWHM-the drop in positron yield in Fig. 2(b) for $\lambda \gtrsim 5 \mu \mathrm{m}$ is an effect of the carrier phase]. This is consistent with a complete calculation from strong-field QED of the pair-creation probability by Meuren et al. [52], which concluded that $P_{ \pm}$scales linearly with $a_{0}$ at constant $\chi$ and approximately linearly with $n$ for $a_{0} \gg 1$.

The positron yield predicted by Eq. (4) always increases with the laser amplitude $a_{0}$, the pulse length $n$, and the photon energy $\omega$. Of these three, it is the amplitude (or peak intensity) that is the most important as there is a dependence on $a_{0}$ in the prefactor and within the nonlinearly increasing function $\mathcal{R}\left(\chi_{\gamma}\right)$. 

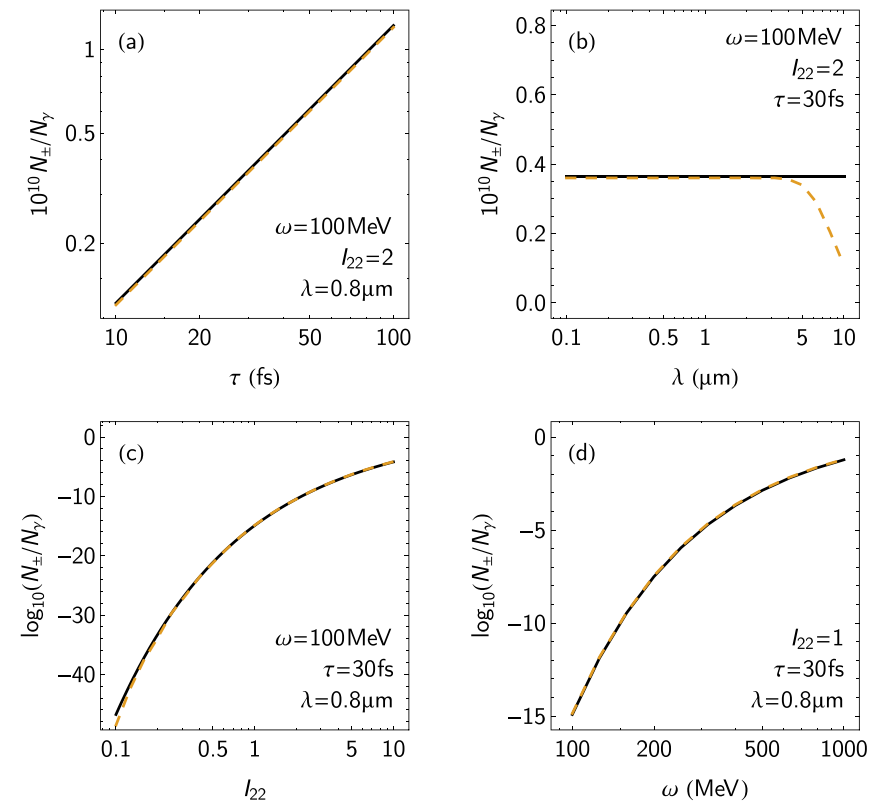

FIG. 2. Number of positrons, $N_{+}$, produced in a collision between a beam of $N_{\gamma}$ photons with energy $\omega$ and a linearly polarized laser pulse that has peak intensity $I_{22} \times 10^{22} \mathrm{~W} \mathrm{~cm}^{-2}$, wavelength $\lambda$, and FWHM $\tau$. Results from our scaling law in Eq. (4) (black) are compared with numerical integration of the full pair-creation rate (dashed yellow).

At the high laser intensities necessary to probe QED effects, one method to explore positron generation which guarantees overlap between the seed photons and the laser pulse is to use the following two-stage process. First, a high-energy electron beam is collided with the pulse in order to generate high-energy photons (within the pulse) by nonlinear inverse Compton scattering. The generated photons can then go on to create pairs by interaction with the laser. In order to estimate the number of positrons produced in this configuration, we now discuss a scaling law for the spectrum of photons produced in nonlinear Compton scattering.

\section{III. $\gamma$-RAY PRODUCTION BY AN ELECTRON BEAM}

\section{A. The effective center of the laser pulse}

As the electron propagates through the intense laser pulse it loses energy through the emission of radiation. When $\chi \gtrsim 0.1$ this emission must be treated quantum mechanically, as then the energy of a single photon can be a significant fraction of the electron energy. The following quantum corrections must be included for our results to be predictive: the reduction in the average radiated power by a factor $g(\chi)$ [64], the explicit form of which is given below, and the stochasticity of the emission process $[28,65,66]$. The former arises because classical theory fails to preclude the emission of photons with more energy than the electron; correcting the shape of the spectrum to guarantee $\omega<\gamma m$ alters the scaling of the radiated power from $\chi^{2}$ to $\chi^{2} g(\chi)$.

Here we consider typical behavior, in the sense that the energy loss, $\gamma$ spectra, and positron yields we predict always implicitly refer to those quantities averaged over an ensemble

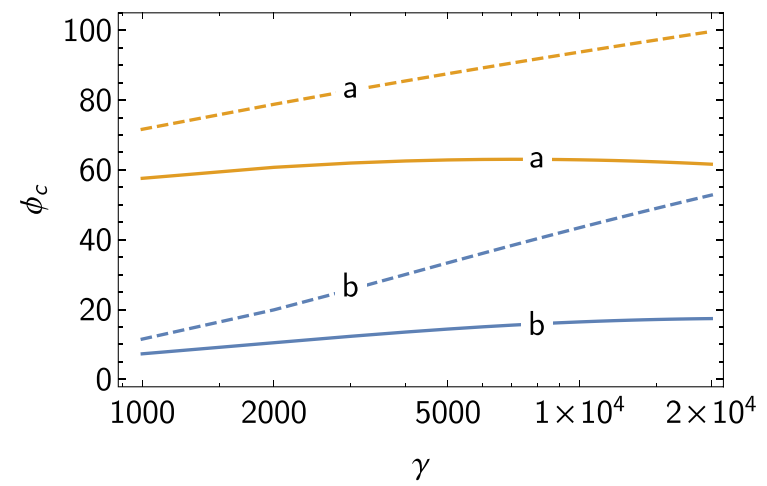

FIG. 3. $\phi_{c}$, the phase at which $\chi$ is maximized, as given by Eqs. (7) and (8) for electrons colliding with laser pulses that have FWHM $30 \mathrm{fs}$, wavelength $800 \mathrm{~nm}(1.55 \mathrm{eV})$, and peak intensity (a) $1 \times 10^{23} \mathrm{~W} \mathrm{~cm}^{-2}$ (yellow) and (b) $1 \times 10^{21} \mathrm{~W} \mathrm{~cm}^{-2}$ (blue). Solid lines are calculated including $g(\chi)$; dashed lines have been calculated in the classical limit $g(\chi)=1$.

of electrons with the same initial $\gamma$. Therefore, the most important of the two corrections is the factor $g(\chi)$, and we adopt a semiclassical approach with a modified, but deterministic, equation of motion. Furthermore, we neglect energy gain from the laser fields, requiring $\gamma \gg a_{0}$, such that the evolution of the electron $\gamma(\phi)$ is determined only by radiative losses:

$$
\frac{d \gamma}{d \phi}=\frac{\mathcal{P}}{m}=\frac{\alpha m \chi^{2} g(\chi)}{3 \omega_{0}},
$$

where $\mathcal{P}$ is the power radiated per unit phase and $g(\chi) \simeq$ $\left[1+4.8(1+\chi) \ln (1+1.7 \chi)+2.44 \chi^{2}\right]^{-2 / 3}[62]$.

As $\chi \propto|\sin \phi|$, Eq. (6) contains an overall fluctuating factor $\sin ^{2} \phi$. The most important phase dependence is the envelope, so we average over this fast oscillation, introducing an overall factor of $\frac{1}{2}$ into Eq. (6). Hereafter, $\chi$ refers to the envelope of the electron's $\chi(\phi)$ such that

$$
\chi=\frac{2 \gamma(\phi) a_{0} \omega_{0}}{m} \exp \left(-\frac{\ln (2) \phi^{2}}{2 \pi^{2} n^{2}}\right) .
$$

Differentiating Eq. (7) with respect to $\phi$ lets us determine the phase $\phi_{c}$ at which $\chi$ is maximized. This will prove particularly significant, as it is where the radiated power is greatest and where the highest-energy photons are emitted. Let $\chi_{c} \equiv \chi\left(\phi_{c}\right)$, which satisfies the following closed relation:

$$
\left[\chi_{c}^{2} g\left(\chi_{c}\right)\right]^{2}=\frac{72 \ln (2)}{\pi^{2} \alpha^{2}}\left(\frac{\gamma \omega_{0}}{n m}\right)^{2} \ln \left(\frac{2 \gamma a_{0} \omega_{0}}{m \chi_{c}}\right) .
$$

This defines $\phi_{c}$ through Eq. (7). It seems we have made little progress though, as both $\chi_{c}$ and $\phi_{c}$ carry a dependence on $\gamma$, the electron energy at $\phi_{c}$, which we do not know a priori. However, the presence of the correction factor $g(\chi)$ means that Eq. (8) has a remarkable property: to a good approximation, it implies that $\chi_{c}$ scales linearly with $\gamma$ and therefore that $\phi_{c}$ is independent of $\gamma$. In other words, $\phi_{c}$ depends only upon the laser pulse parameters.

In Fig. 3 we show the $\phi_{c}$ predicted by Eqs. (7) and (8) for various $\gamma$ and fixed laser pulses. It does appear that $\phi_{c}$ is independent of the chosen $\gamma$ to a good approximation. To 

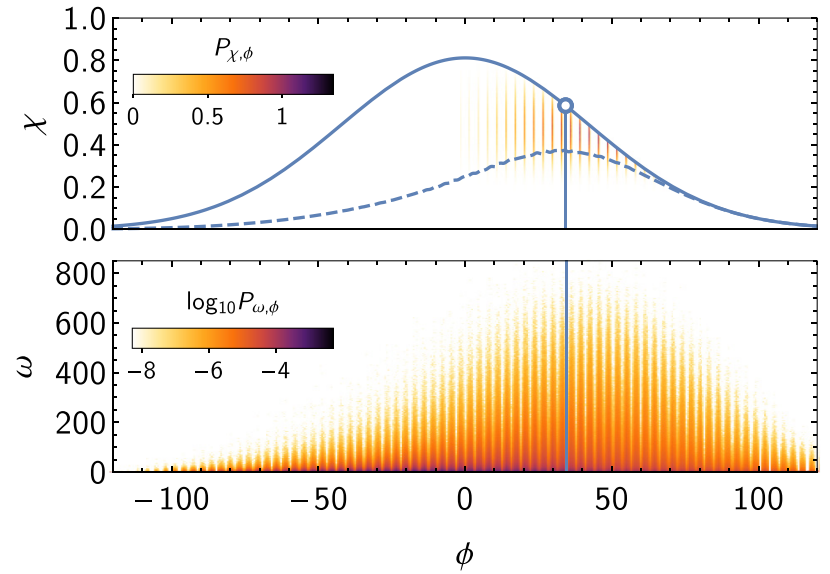

FIG. 4. Top: Color scale, the probability density $P_{\chi, \phi}$ that a stochastically radiating electron reaches a maximum quantum parameter $\chi$ at phase $\phi$; solid blue curve, the $\chi$ of an electron that loses no energy; dashed blue curve, the $\chi$ of an electron that loses energy according to Eq. (6); and circle, the $\chi_{c}$ and $\phi_{c}$ given by Eqs. (7) and (8). Observe that the region of maximum emission probability is correctly identified by the predicted $\phi_{c}$. Bottom: Color scale, the probability density $P_{\omega, \phi}$ that a photon is emitted with energy $\omega$ at phase $\phi$ and (vertical line) $\phi_{c}$. (See text for collision parameters.)

demonstrate that the origin of this effect is the inclusion of $g(\chi)$, we also show $\phi_{c}$ for the classical condition $g(\chi)=1$. In this case, by contrast, $\phi_{c}$ increases with increasing $\gamma$. Let us justify this phenomenon by differentiating Eq. (8) with respect to $\gamma$ to study the quantity $\partial \ln \chi_{c} / \partial \ln \gamma$. We find that

$$
\left[\frac{2}{\chi_{c}}+\frac{\partial \ln g\left(\chi_{c}\right)}{\partial \chi_{c}}\right] \frac{\partial \chi_{c}}{\partial \ln \gamma}-1=\frac{A \gamma^{2}}{2 \chi_{c}^{4} g\left(\chi_{c}\right)^{2}}\left(1-\frac{\partial \ln \chi_{c}}{\partial \ln \gamma}\right),
$$

where $A=72 \ln (2) \omega_{0}^{2} /(\pi \alpha n m)^{2}$ is the coefficient of $\gamma^{2}$ on the right-hand side (RHS) of Eq. (8). The factor in square brackets on the left-hand side (LHS) of the above relation bears study. Classically (or, equivalently, in the limit $\chi_{c} \ll 1$ ) it becomes $2 / \chi_{c}$; combining this with the fact that $A \ll 1$ we find that $\chi_{c}$ scales approximately as $\gamma^{1 / 2}$ for $\chi_{c} \ll 1$. However, for $\chi_{c} \gtrsim 0.1$, it is very close to $1 / \chi_{c}$ and therefore $\chi_{c} \propto \gamma$, giving $d \phi_{c} / d \phi \simeq 0$ as suggested in Fig. 3 .

This linear scaling only holds for "reasonable" values of $\chi_{c}$, but we emphasize that because our results depend on the locally constant field and rigid-beam approximations, we begin by assuming $\gamma \gg a_{0} \gg 1$, and so for all realistic laser-electronbeam collisions we have $\chi \gtrsim 0.1$. We may therefore replace $\gamma$ in Eqs. (7) and (8) with $\gamma_{0}$, the Lorentz factor of the electron before the collision. $\phi_{c}$ is still the phase where $\chi$ is maximized and $\chi_{c}$ becomes the $\chi$ of an electron that has reached that phase without losing energy. This is possible in the quantum radiation reaction regime due to straggling (quenching) [65,66].

Figure 4 compares the predicted $\phi_{c}$ and $\chi_{c}$ to the results of a single-particle Monte Carlo simulation of quantum radiation reaction. The initial energy of the electron is $1 \mathrm{GeV}$ and the laser pulse has wavelength $0.8 \mu \mathrm{m}$, FWHM $30 \mathrm{fs}$, and peak intensity $1 \times 10^{22} \mathrm{~W} \mathrm{~cm}^{-2}\left(\gamma_{0}=1957, \omega_{0}=1.55 \mathrm{eV}\right.$, $n=11.2$, and $a_{0}=68.3$ ). For each electron we track the maximum $\chi$ experienced along its trajectory, as well as the phase at which this occurred. The probability density $P_{\chi, \phi}$ that an electron reaches $\chi$ at phase $\phi$ is plotted in the upper panel of Fig. 4; to aid the eye, the $\chi(\phi)$ of a nonradiating and semiclassically radiating electron are plotted as well. We see that $\phi_{c}$ accurately captures the point at which the electron $\chi$ is maximized, in both the semiclassical and the stochastic case. Comparison with the probability density $P_{\omega, \phi}$ that a photon is emitted with energy $\omega$ at phase $\phi$, plotted in the lower panel, shows that $\phi_{c}$ also characterizes the region where the highest-energy photons are emitted.

\section{B. Energy loss of the electron beam}

We now derive a scaling law for the energy loss of an electron in the quantum-radiation-reaction-dominated regime. Of course, we could simply solve Eq. (6) given the pulse parameters, but as $g(\chi)$ has no simple analytical form, those results would necessarily be numerical. Instead we use the results of Sec. III A to guide us toward an approximate, but analytical, scaling law. We expect that $\chi$ and the radiated power are strongly peaked in the region around $\phi=\phi_{c}$, so we use the Laplace method (i.e., the saddle-point approximation) to estimate the radiated energy in the absence of recoil. Then we employ the single-photon recoil correction $\Omega \rightarrow \Omega /(\gamma m-$ $\Omega$ ) to obtain a recoil-corrected estimate of both $\chi_{c}$ and the final electron energy.

The Laplace method for the integral $\int \mathcal{P}(\phi) d \phi$ effectively replaces the integrand with a Gaussian with peak $\mathcal{P}_{c}$ and variance $\sigma^{2}=-\mathcal{P}_{c} / \mathcal{P}_{c}^{\prime \prime}$, these being evaluated at the point $\phi_{c}$ where $\mathcal{P}^{\prime}$ vanishes. Here primes denote differentiation with respect to $\phi$. Then the integral is $\left[2 \pi \mathcal{P}_{c}^{3} /\left(-\mathcal{P}_{c}^{\prime \prime}\right)\right]^{1 / 2}$. We have that

$$
\mathcal{P}_{c}=\frac{\alpha m^{2} \chi_{c}^{2} g\left(\chi_{c}\right)}{6 \omega_{0}}
$$

using the results of Sec. III A. The second derivative

$$
\mathcal{P}_{c}^{\prime \prime}=\left.\mathcal{P}_{c}\left[\frac{2}{\chi_{c}}+\frac{\partial \ln g\left(\chi_{c}\right)}{\partial \chi_{c}}\right] \chi^{\prime \prime}\right|_{\phi=\phi_{c}},
$$

where

$$
\left.\chi^{\prime \prime}\right|_{\phi=\phi_{c}}=-\frac{\ln (2) \chi_{c}}{\pi^{2} n^{2}}\left[1+2 \ln \left(\frac{2 \gamma_{0} a_{0} \omega_{0}}{m \chi_{c}}\right)\right] .
$$

Equation (11) contains the same factor in square brackets as Eq. (9); as before, we replace it with $1 / \chi_{c}$. Then we find that the radiated energy (in the absence of recoil) is

$$
\Omega=\sqrt{2 \pi} \gamma_{0} m\left[\frac{2 \ln \left(\frac{2 \gamma_{0} a_{0} \omega_{0}}{m \chi_{c}}\right)}{1+2 \ln \left(\frac{2 \gamma_{0} a_{0} \omega_{0}}{m \chi_{c}}\right)}\right]^{1 / 2} .
$$

The argument of the logarithms is always $\geqslant 1$; the equality would correspond to $\phi_{c}=0$ and $\chi_{c}$ taking on its largest possible value at the pulse center. Therefore, $0 \leqslant \Omega \leqslant \sqrt{2 \pi} \gamma_{0} m$.

Let $\Omega_{\mathrm{rr}}$ be the total energy emitted in photons when we do account for the electron recoil, i.e., radiation reaction. Were we to assume that only one photon is emitted, the firstorder correction would give $\Omega_{\mathrm{rr}}=\Omega /\left(1+\Omega / \gamma_{0} m\right)[67,68]$. However, as the electron emits many photons, this is not a very good approximation. We are guided instead by the fact that the radiated energy should be approximately symmetric around 
the point $\phi=\phi_{c}$. This is exactly true for the Laplace method because the fitted Gaussian is centered at $\phi=\phi_{c}$. It will still be true after we account for recoil because $\phi_{c}$ is independent of $\gamma$ and must still mark the point of maximum radiated power. We have, by our argument for the symmetry of the radiated energy, that the electron loses $\Omega / 2$ during the interval $\phi>\phi_{c}$; therefore, its Lorentz factor and recoil-corrected $\chi$ at $\phi_{c}$ satisfy

$$
\frac{\gamma_{c}}{\gamma_{0}}=\frac{\chi_{c, \mathrm{rr}}}{\chi_{c}} \simeq\left(1+\frac{\Omega}{2 \gamma_{0} m}\right)^{-1} .
$$

Repeating the process for the interval $\phi<\phi_{c}$, the electron final Lorentz factor is then

$$
\gamma_{\mathrm{f}} \simeq \frac{2 \gamma_{0} m-\Omega}{2 \gamma_{0} m+\Omega} \gamma_{0} .
$$

This is positive only if $\Omega<2 \gamma_{0} m$; as we saw earlier, $\Omega$ is bounded by $\sqrt{2 \pi} \gamma_{0} m \simeq 2.51 \gamma_{0} m$. Nevertheless, as we seek a scaling law for the photon spectrum, it is more important that both $\gamma_{c}$ and $\chi_{c, \text { rr }}$ are correctly bounded by zero from below, which they indeed are.

We can also estimate the electron Lorentz factor and $\chi$ as a function of phase, using that the radiated power and $\chi$ as functions of phase are approximately Gaussian in form:

$$
\begin{gathered}
\gamma(\phi) \simeq \gamma_{\mathrm{f}}+\frac{\gamma_{0} \Omega}{2 \gamma_{0} m+\Omega}\left[1+\operatorname{erf}\left(\frac{\phi-\phi_{c}}{\sqrt{2} \sigma}\right)\right], \\
\chi(\phi) \simeq \frac{\chi_{c}}{1+\frac{\Omega}{2 \gamma_{0} m}} \exp \left(-\frac{\left(\phi-\phi_{c}\right)^{2}}{2 \sigma^{2}}\right),
\end{gathered}
$$

where

$$
\sigma^{2}=\frac{\pi^{2} n^{2}}{\ln (2)}\left[1+2 \ln \left(\frac{2 \gamma_{0} a_{0} \omega_{0}}{m \chi_{c}}\right)\right]^{-1} .
$$

Comparison between Eq. (17) and numerical solutions to the equation of motion [Eq. (6)] are given in Fig. 5, for experimental parameters corresponding to Gemini [Fig. 5(a)], the Berkeley Lab Laser Accelerator (BELLA) [Fig. 5(b)], SLAC [Fig. 5(c)], and ELI [Fig. 5(d)]. There is excellent agreement with respect to the maximum $\chi$, the phase at which it is reached, and the distance over which it is sustained. This demonstrates the importance of accounting for radiation reaction, as without doing so we would overestimate $\chi$.

\section{Photon energy spectrum}

Figure 4 confirms that the region near $\phi_{c}$, where $\chi$ is maximized, is the origin of the highest-energy photons. We propose that the spectrum may be approximated by assuming that the electron has $\chi$ as given by Eq. (17) and energy $\gamma \simeq \gamma_{0}$ at this point; the latter is our way of accounting for straggling (quenching). As we are interested in the high-energy tail of the spectrum, we expand the double differential rate of emission for $\chi_{\gamma} \sim \chi$ :

$$
\frac{\partial^{2} N_{\gamma}}{\partial t \partial \chi_{\gamma}} \simeq \frac{\alpha m}{2 \sqrt{\pi} \gamma} \frac{\exp \left(-\frac{2 \chi_{\gamma}}{3 \chi\left(\chi-\chi_{\gamma}\right)}\right)}{\sqrt{\chi-\chi_{\gamma}}}
$$

which may be converted into an integral over phase and photon energy using that $\phi=-2 \omega_{0} t$ and $\omega /(\gamma m)=\chi_{\gamma} / \chi$. Then we use the Laplace method again with Eqs. (12) and (17), with

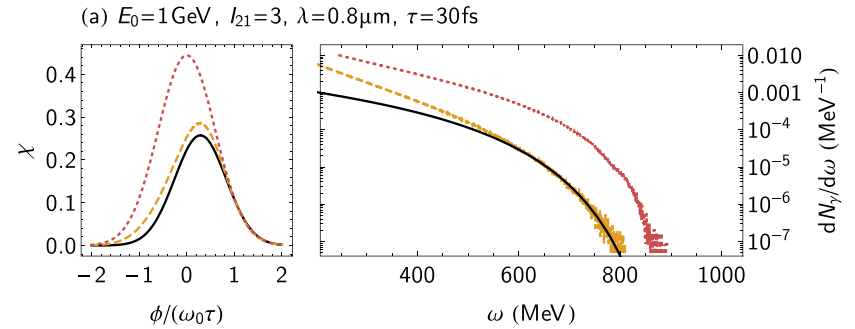

(b) $E_{0}=4 \mathrm{GeV}, l_{21}=0.8, \lambda=0.8 \mu \mathrm{m}, \tau=40 \mathrm{fs}$
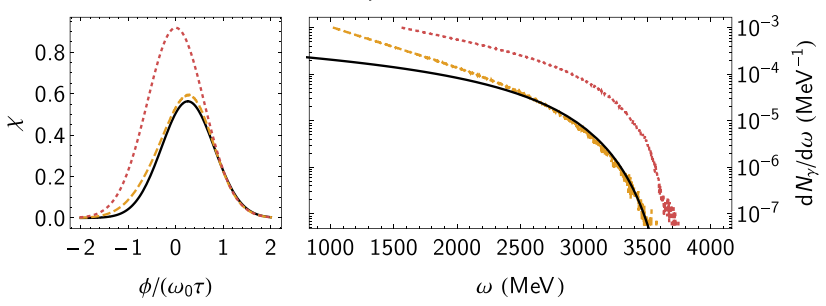

(c) $E_{0}=50 \mathrm{GeV}, l_{21}=0.1, \lambda=1 \mu \mathrm{m}, \tau=100 \mathrm{fs}$

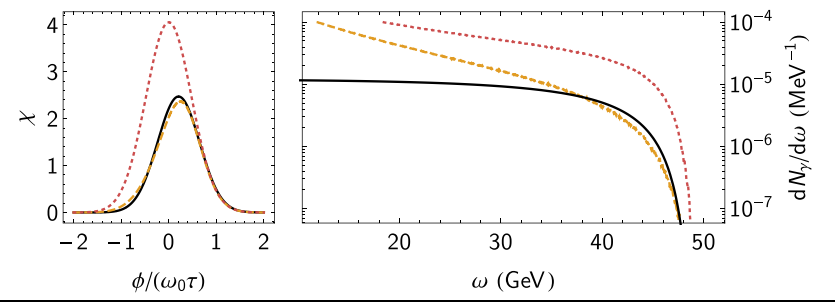

(d) $E_{0}=4 \mathrm{GeV}, I_{21}=100, \lambda=0.8 \mu \mathrm{m}, \tau=10 \mathrm{fs}$
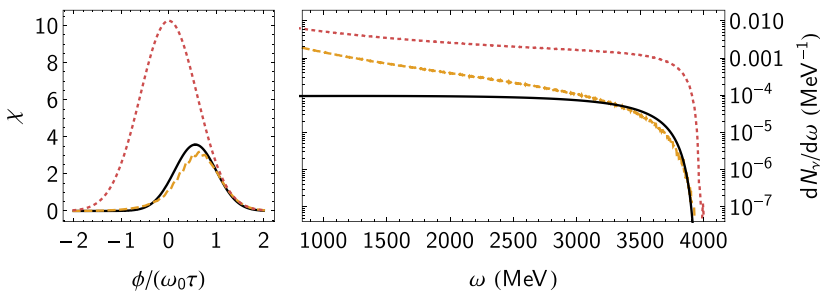

FIG. 5. For a collision between an electron beam with energy $E_{0}$ and a linearly polarized laser pulse with peak intensity $I_{21} \times$ $10^{21} \mathrm{~W} \mathrm{~cm}^{-2}$, wavelength $\lambda$, and FWHM $\tau$, left, the electron quantum nonlinearity parameter $\chi$ as a function of phase $\phi$, as predicted by Eq. (17) (dashed yellow curves), solution to equation of motion [Eq. (6)], and in the absence of radiation reaction (dotted red curves); right, energy spectra (normalized per electron) of the emitted photons, as predicted by our scaling [Eq. (20), solid black curves] and Monte Carlo simulation with stochastic radiation reaction (dashed yellow curves) and no radiation reaction (dotted red curves).

the result that

$$
\begin{aligned}
\frac{d N_{\gamma}}{d \omega} \simeq & \frac{\sqrt{3} \pi \alpha F_{\mathrm{he}}}{\sqrt{2 \ln (2)}} \frac{a_{0} n}{\sqrt{E_{0}}} \frac{\chi_{c, \mathrm{rr}} / \chi_{0}}{\sqrt{1+2 \ln \left(\chi_{0} / \chi_{c}\right)}} \\
& \times \frac{\exp \left(-\frac{2 \omega}{3 \chi_{c, \mathrm{rr}}\left(E_{0}-\omega\right)}\right)}{\sqrt{3 \chi_{c, \mathrm{rr}}\left(E_{0}-\omega\right)+4 \omega}}
\end{aligned}
$$

for $E_{0}=\gamma_{0} m, \chi_{0}=2 \gamma_{0} a_{0} \omega_{0} / m$, and $\chi_{c, \text { rr }}$ related to $\chi_{c}$ via Eq. (14). Now, as not every emission qualifies as "high energy," this overestimates the number of hard photons. To account for this we multiply Eq. (20) by a correction factor $F_{\text {he }}$ which is the ratio of the number of photons emitted for $\phi>\phi_{c}$ to the 
total number of photons:

$$
F_{\text {he }}=\frac{1}{2}\left[1-\operatorname{erf}\left(\frac{\sqrt{2 \ln (2)} \phi_{c}}{2 \pi n}\right)\right] .
$$

This works because, as we showed in Sec. III B, the electron loses most of its energy for $\phi \simeq \phi_{c}$; only for larger phases can it be said still to be "high energy."

We compare the predicted scalings with simulation data in Fig. 5 (for consistency, pair creation and therefore secondary photon emission were disabled). The logarithmic scaling of the vertical axes admittedly flatters the comparison, but we find good agreement between the stochastic data and our simple scaling. It captures the shape of the high-energy tail, the absolute number of photons, and the reduction in both caused by radiation reaction.

\section{POSITRON PRODUCTION BY AN ELECTRON BEAM}

\section{A. Number of pairs}

The number of positrons produced by a laser-electronbeam collision may be determined by convolving the paircreation probability [Eq. (4)] with the photon spectrum [Eq. (20)]. Strictly, this requires that the contribution to the probability $P_{ \pm}$from the region $\phi>\phi_{c}$ is negligible, as the daughter photon beam is actually created within the laser pulse near $\phi=\phi_{c}$.

While the pair-creation probability is always (nonlinearly) increasing with increasing photon energy, the photon number is always decreasing because of the exponential factor in Eq. (20). Therefore, the probability spectrum $P_{ \pm} \frac{d N_{\gamma}}{d \omega}$ is peaked for some $\omega_{c}<\gamma_{0} m$. (If one considers the ensemble of positrons emerging from the laser-beam collision and looks at the energy distribution of their parent photons, $\omega_{c}$ will be most probable.) Let us consider the threshold regime for pair creation, which is currently accessible, where the yield is dominated by the conversion of the highest-energy photons. Then we may expect the combination of our scaling laws for the photon spectrum and pair probability to be predictive.

First we derive a relation for $\omega_{c}$. Let $S(\omega)$ be the last factor in Eq. (20), the only part that depends on the photon energy. Then the product $P_{ \pm} \frac{d N_{\gamma}}{d \omega}$ is maximized for $\omega=\omega_{c}$ satisfying

$$
\frac{\mathcal{R}^{\prime}\left(\chi_{\gamma}\right)}{\mathcal{R}\left(\chi_{\gamma}\right)} \frac{\partial \chi_{\gamma}}{\partial \omega}=\frac{\left|S^{\prime}\left(\omega_{c}\right)\right|}{S\left(\omega_{c}\right)},
$$

where $\chi_{\gamma}=2 a_{0} \omega_{0} \omega_{c} / m^{2}$ and $\mathcal{R}$ was introduced in Eq. (5). We expect $\omega_{c}$ to be near $E_{0}$, so we take only the leadingorder term in $\left(\gamma_{0} m-\omega\right)$ from the RHS. The LHS depends on the properties of $\mathcal{R}$ but we find that for $\chi_{\gamma} \leqslant 10, \mathcal{R}^{\prime} / \mathcal{R} \simeq$ $2.75 / \chi_{\gamma}^{2}$. Therefore, we have

$$
\omega_{c} \simeq \gamma_{0} m \frac{\sqrt{\frac{2 \chi_{c, \mathrm{r} m} m}{a_{0} \gamma_{0} \omega_{0}}}}{1+\sqrt{\frac{2 \chi_{c, \mathrm{rr}} m}{a_{0} \gamma_{0} \omega_{0}}}} .
$$

We use this point as the origin of a saddle-point approximation to the integral $\int P_{ \pm} \frac{d N_{\gamma}}{d \omega} d \omega$, which gives us the positron yield arising from a high-energy electron beam. We take only the leading-order term in $\left(\gamma_{0} m-\omega_{c}\right)$ as before. Leaving out the details, we find that the number of positrons produced per
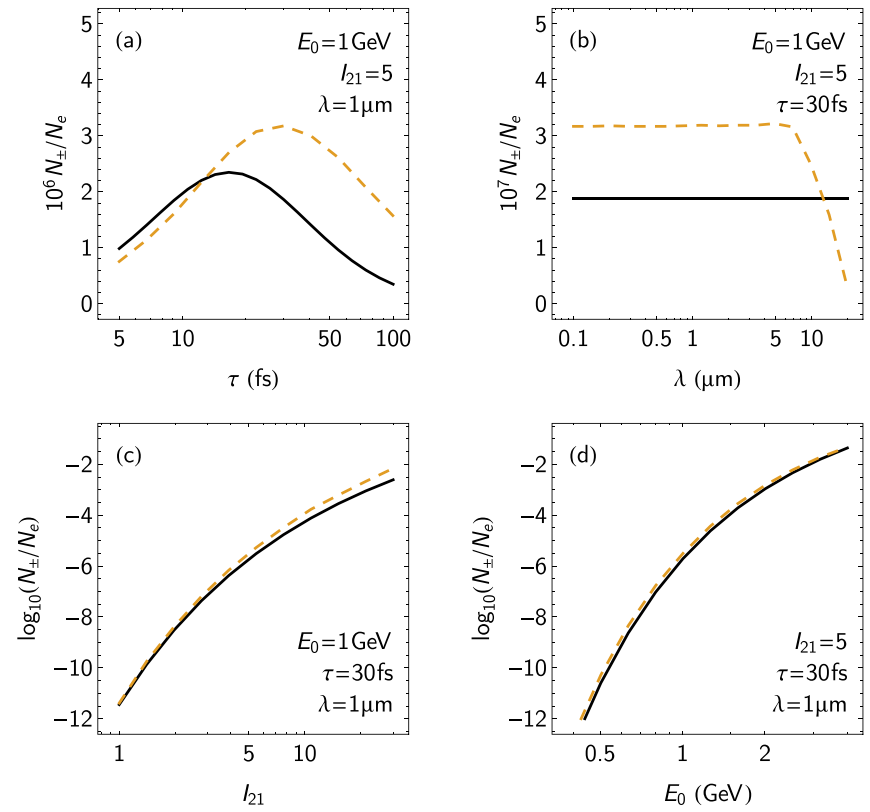

FIG. 6. Number of positrons, $N_{+}$, produced in a collision between a beam of $N_{e}$ electrons with energy $E_{0}$ and a linearly polarized laser pulse that has peak intensity $I_{21} \times 10^{21} \mathrm{~W} \mathrm{~cm}^{-2}$, wavelength $\lambda$, and FWHM $\tau$. Results from our scaling law [Eq. (24), solid black curve] and simulations using the full pair-creation rate (dashed yellow curve).

electron is

$$
\left.N_{+} \simeq \frac{3 \sqrt{\pi} P_{ \pm}\left(\omega_{c}\right) \chi_{c, \mathrm{rr}}}{\sqrt{2}} \frac{\left(\gamma_{0} m-\omega_{c}\right)^{2}}{\gamma_{0} m} \frac{d N_{\gamma}}{d \omega}\right|_{\omega=\omega_{c}}
$$

using the recoil-corrected $\chi_{c, \text { rr }}$ from Eq. (14), $P_{ \pm}$from Eq. (4), and the photon spectrum from Eq. (20).

The results of this calculation are compared with the yield obtained from Monte Carlo simulation in Fig. 6, for collision parameters that are within the scope of present-day laser facilities. We find that it is accurate to within a factor of 2 to 3 across the range of explored parameters, with a tendency to underestimate the yield. This is because the approximate spectrum in Eq. (20), while accurate for the high-energy tail, underestimates the number of low- to mid-energy photons. At lower intensities, positron production is dominated by the high-energy tail of the spectrum so our prediction is accurate. As $\chi_{\gamma}$ exceeds 1, pair creation is possible for photons across a wider energy range, and our prediction will undershoot.

Increasing $\gamma$ and $a_{0}$ for fixed pulse length $n$ always increases the positron yield. However, for fixed $\gamma_{0}$ and $a_{0}$, we see from Fig. 6(a) that there is a laser pulse length where the positron yield is maximized. This may be understood by considering the competing factors of $P_{ \pm}$and $\chi_{c \text {,rr }}$ in Eq. (24). The former favors increasing pulse length as photon decay becomes more probable. The latter accounts for the sensitivity of the yield to the pulse rise time (which increases with $n$ ), as increased energy loss of the electron beam in the rising edge suppresses growth of $\chi$ and consequently hard photon emission. This is why our scaling underestimates the yield for $\tau \gtrsim 40$ fs: the photon spectrum in this region is dominated by low- to 
mid-energy photons which the scaling in Eq. (20) does not capture.

\section{B. Mean positron energy}

Now we consider the effect of secondary photon emission on the positron energy. To produce large numbers of pairs we need $\chi_{\gamma} \gtrsim 1$, implying a positron initial $\chi_{+} \gtrsim 0.5$ which lies within the quantum radiation reaction regime. We assume that the positron (equivalently, the electron) is created at $\phi=0$ with half the energy of the parent photon. We take this to be the most probable parent photon energy $\omega_{c}$, as given by Eq. (23). The procedure to determine the final energy of the positron is similar to that outlined in Sec. III B. Both $\chi_{+}$and the radiated power are maximized at $\phi=0$ and decrease with decreasing $\phi$. Given this, we integrate the radiated power from $\phi=-\infty$ to $\phi=0$ using the Laplace method to determine radiated energy without recoil, then apply the single-photon correction. We find that the postcollision Lorentz factor of the positron is

$$
\gamma_{+} \simeq \frac{\omega_{c}}{2 m}\left[1+\frac{\pi^{3 / 2} \alpha}{3 \sqrt{2 \ln (2)}} \frac{n a_{0}^{2} \omega_{0} \omega_{c}}{m^{2}} g\left(\frac{a_{0} \omega_{0} \omega_{c}}{m^{2}}\right)\right]^{-1} .
$$

Comparison with Monte Carlo simulation, shown in Fig. 7, shows that this scaling law accurately predicts the mean positron final energy. Nevertheless, we see it breaking down for $\gamma_{0} m$ larger than a GeV, as the initial positron spectrum for $\chi_{\gamma}>$ 1 , while still symmetric around $m \gamma_{+}=\omega_{c} / 2$, is much broader and stochastic effects are more pronounced. The positron
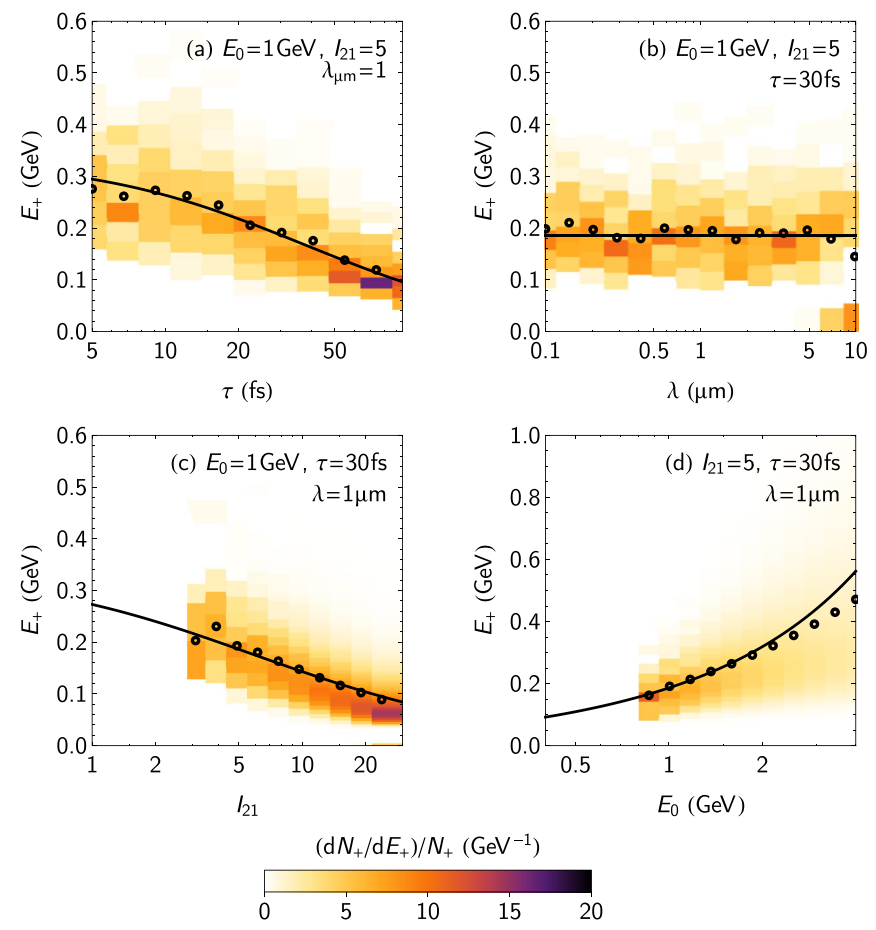

FIG. 7. Color scale, energy spectra of positrons emerging from a collision between an electron beam with energy $E_{0}$ and a laser pulse with peak intensity $I_{21} \times 10^{21} \mathrm{~W} \mathrm{~cm}^{-2}$, wavelength $\lambda$, and FWHM $\tau$; black lines, the characteristic energy predicted by Eqs. (23) and (25); and black circles, the mean energy of the simulated spectra. spectrum is unchanged as the wavelength increases until $n=c \tau / \lambda \gtrsim 2$, at which point carrier-phase effects become significant. The laser pulse we consider has phase dependence $\sin \phi$, so this means the pair creation is switched off as the pulse FWHM shrinks. In general the width of positron spectrum increases with increasing electron-beam energy and decreases with increasing pulse intensity and duration.

\section{Prospects for experimental observation}

We have seen that the optimal pulse length for pair creation from $\mathrm{GeV}$ electron beams and laser pulses with intensity in the high $1 \times 10^{21} \mathrm{~W} \mathrm{~cm}^{-2}$ is approximately $30 \mathrm{fs}$, which is close to the characteristic duration of current high-intensity lasers. Therefore, we show in Fig. 8 the number and characteristic energy of positrons produced in such a collision, taking the wavelength of the light to be $1 \mu \mathrm{m}$ and the FWHM of the pulse to be $30 \mathrm{fs}$. The positron yield increases substantially with both increasing electron-beam energy and laser intensity. Laser wake fields typically accelerate bunches of charge 100 $\mathrm{pC}$, implying that to produce more than 100 positrons in a single shot requires a laser intensity $I_{0}$ and beam energy $E_{0}$ that satisfy

$$
\left(\frac{I_{0}}{1 \times 10^{21} \mathrm{~W} \mathrm{~cm}^{-2}}\right)\left(\frac{E_{0}}{2 \mathrm{GeV}}\right)^{2} \gtrsim 1 .
$$

This condition can be met in present-day high-intensity laser facilities, where we expect measurable quantities of $>100 \mathrm{MeV}$ positrons to be produced. However, to be confident that this is the case, we extend our results to account for the fact that both laser and electron beam have finite size.

Consider a beam of $N_{e, \mathrm{~b}}$ electrons with spherically symmetric Gaussian charge density ( $\operatorname{size} R$ ), offset from the laser axis by a distance $\Delta$. Without loss of generality we may choose that offset to be in the $x$ direction. The peak laser intensity each electron encounters depends upon that electron's spatial and temporal offset from the laser focus. Let $(x, y)$ be the position at which an individual electron encounters the peak of the laser pulse. Then the effective $a_{0}$ of the pulse for that electron becomes

$$
a \simeq a_{0} \exp \left(-\frac{x^{2}+y^{2}}{w_{0}^{2}}\right),
$$

where $w_{0}$ is the laser waist. The number of electrons that encounter the pulse peak at $(x, y)$, experiencing an effective $a_{0}$ given by Eq. (27), is

$$
d N_{e}=\frac{N_{e, \mathrm{~b}}}{\pi R^{2}} \exp \left(-\frac{(x-\Delta)^{2}+y^{2}}{R^{2}}\right) d x d y .
$$

The total number of positrons produced by a beam is $N_{+, \mathrm{b}}=$ $\int N_{+}(x, y) d N_{e}$, where $N_{+}(x, y)$ is obtained by replacing the $a_{0}$ in Eq. (24) with $a$ as given by Eq. (27).

We take as an example the collision between a $2-\mathrm{GeV}$ electron beam (total charge $100 \mathrm{pC}$, spherically symmetric FWHM $10 \mu \mathrm{m}, R=6 \mu \mathrm{m}$ ) and laser pulse with peak intensity $5 \times 10^{21} \mathrm{~W} \mathrm{~cm}^{-2}$, wavelength $0.8 \mu \mathrm{m}$, FWHM $30 \mathrm{fs}$, and waist $2 \mu \mathrm{m}$ and compare our predictions to the result of full-scale three-dimensional (3D) PIC simulation (see Appendix B for details). Were we to take this as a plane-wave interaction, we 

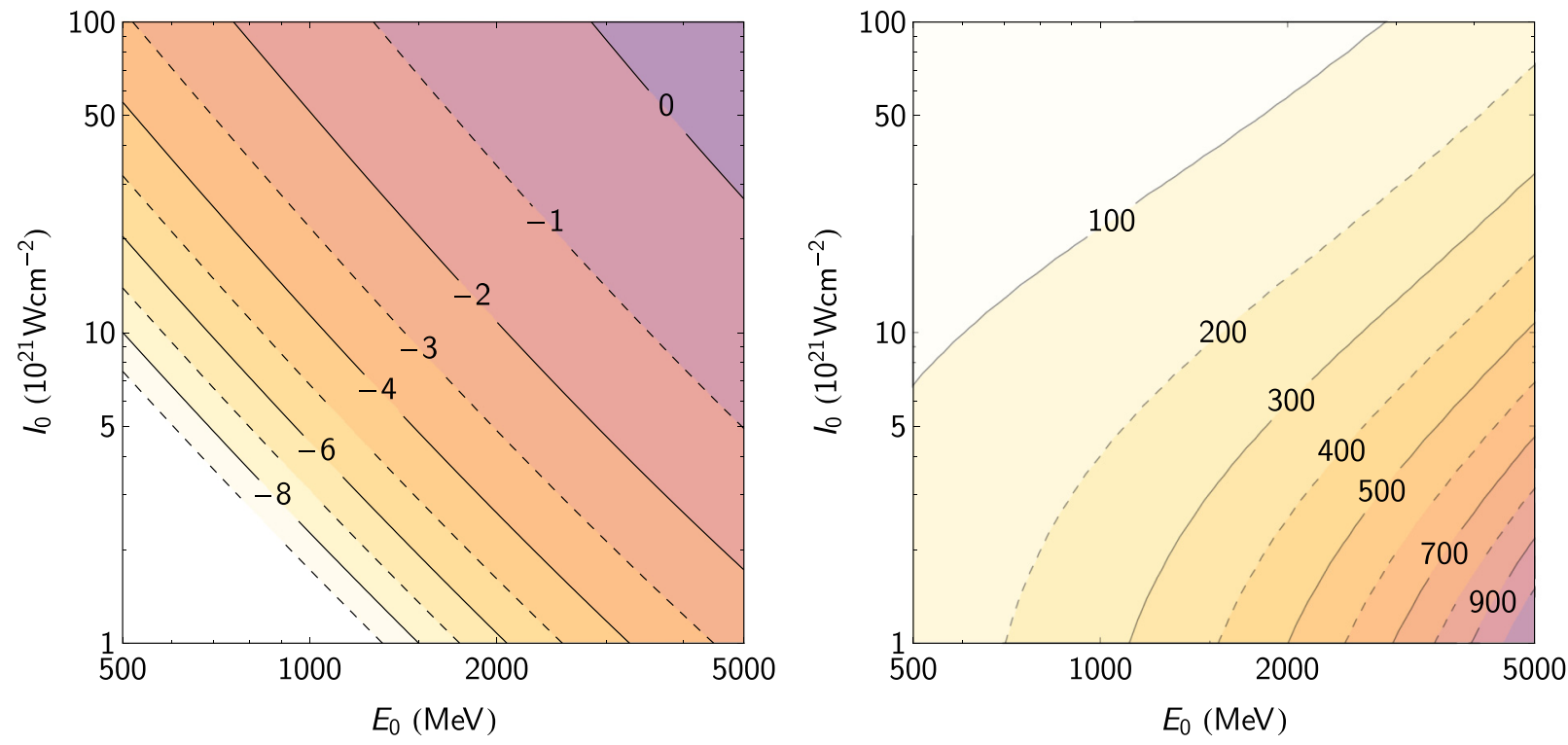

FIG. 8. Left: $\log _{10}$-scaled yield (per electron). Right: Typical energy in $\mathrm{MeV}$ of positrons produced in the collision of an electron beam with energy $E_{0}$ and a laser pulse with peak intensity $I_{0}$, wavelength $1 \mu \mathrm{m}$, and FWHM 30 fs, as predicted by Eq. (24).

would predict a positron yield of $6.9 \times 10^{5}$ using Eq. (24). Accounting for beams' finite sizes by integrating $\int N_{+}(x, y) d N_{e}$ numerically using Eqs. (24) and (27), we find $N_{+, b} \simeq 10500$. This is in good agreement with $N_{+, b} \simeq 10000$ from simulation and should be assessed in light of the nonperturbative growth in the pair-creation probability shown in Fig. 8. We find also that the final energy of the positron beam predicted by Eqs. (25) and (23), $320 \mathrm{MeV}$, is consistent with the spectrum shown in Fig. 9. With the addition of a perpendicular offset of 5 $\mu \mathrm{m}$ between the beams, numerical evaluation predicts that the positron yield should be reduced to 5300; we find $N_{+, b}=4700$ in PIC simulation.

To provide an approximate analytical scaling for the number of positrons produced by an electron beam of finite size, we evaluate the integral $\int N_{+}(x, y) d N_{e}$ with the Laplace method, assuming that $R \gg w_{0}$ and that the dominant contribution is

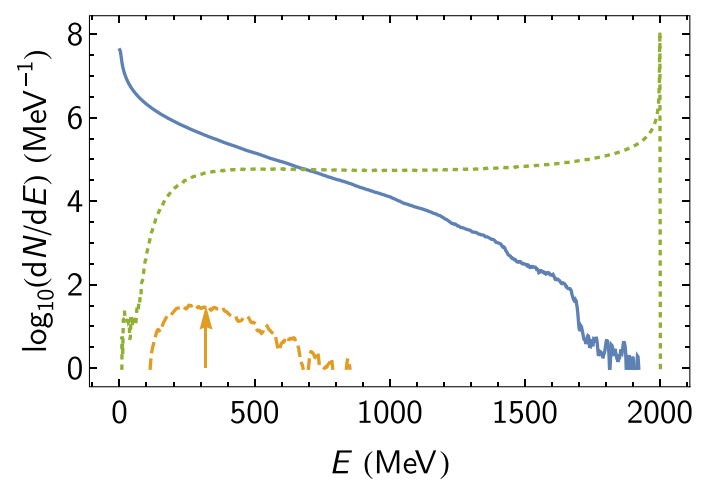

FIG. 9. The energy spectra of photons (solid blue curve), positrons (dashed yellow curve), and beam electrons (dotted green curve) emerging from a collision between an electron beam with energy $2 \mathrm{GeV}$ and a laser pulse with peak intensity $5 \times 10^{21} \mathrm{~W} \mathrm{~cm}^{-2}$ (see Sec. IV C for details). The positron energy predicted by Eqs. (25) and (23) is indicated by a yellow arrow. that of the region near $x=y=0$ where the field amplitude is largest. The fastest dependence on $a$ (and therefore $x, y$ ) in Eq. (24) is that of the auxiliary function $\mathcal{R}$, so we keep all other factors constant when evaluating the Hessian of $N_{+}(x, y)$. We find the number of positrons produced by an $R$-radius beam of $N_{e, \mathrm{~b}}$ electrons colliding with a laser pulse of waist $w_{0}$ with perpendicular offset $\Delta$ to be

$$
N_{+, \mathrm{b}} \simeq \frac{0.727 a_{0} \omega_{0} \omega_{c}}{m^{2}} \frac{w_{0}^{2} e^{-\Delta^{2} / R^{2}}}{R^{2}} N_{+} N_{e, \mathrm{~b}}
$$

for $w_{0}<R$ and $N_{+}$given by Eq. (24). The leading factor may be taken to be roughly 0.25 , because, to have substantial pair creation at all, $\omega_{c}$ must be sufficiently large that $2 a_{0} \omega_{0} \omega_{c} / m^{2} \gtrsim 1$. For the collision parameters given above, Eq. (29) predicts $N_{+, b} \simeq 18600$ and 9300 for an offset of 0 and $5 \mu \mathrm{m}$ respectively, accurate to within a factor of 2 .

Equation (29) indicates that the accuracy of alignment between the electron beam and the laser pulse must be about the size of the electron beam itself. It suggests further that it is advantageous to focus the laser pulse as tightly as possible, increasing $a_{0}$ at the expense of $w_{0}$. The latter only enters the scaling quadratically, whereas $N_{+}$grows much faster with $a_{0}$ through its dependence on $\mathcal{R}$ [Eq. (5)]. Analytical work on the effect of tight focusing has already begun [54,55], going beyond the plane-wave approximation to explore the effect of wave-front curvature on the positron yield. Nevertheless, as near-term experiments are likely to focus the intense laser with optics with $f$ number closer to 2 , the effects of finite size and alignment errors are more significant.

\section{SUMMARY}

The collision of an intense laser pulse with a high-energy electron beam is a promising experimental geometry for the production of high-energy photons and positrons. We have presented analytical expressions for the electron beam's energy loss, quantum nonlinearity parameter, and self-consistent 
emission spectrum. Our scaling law for the number of positrons produced by the emitted $\gamma$ rays demonstrates good agreement with full-scale PIC simulation even when the finite sizes of the colliding beams are accounted for. We have shown that a near-term experiment employing the collision of a $2-\mathrm{GeV}$ electron beam and laser pulse of intensity $5 \times 10^{21} \mathrm{~W} \mathrm{~cm}^{-2}$ will produce a positron beam with energy $300 \mathrm{MeV}$ and particle number $1 \times 10^{4}$. Experimental detection of this beam will provide unambiguous evidence of pair creation via the nonlinear Breit-Wheeler process.

\section{ACKNOWLEDGMENTS}

The authors acknowledge support from the Knut and Alice Wallenberg Foundation (T.G.B. and M.M.), the Swedish Research Council (Grants No. 2012-5644 and No. 2013-4248, M.M.), and the European Union's Horizon 2020 research and innovation program under the Marie Skłodowska-Curie Grant No. 701676 (A.I.). Simulations were performed on resources provided by the Swedish National Infrastructure for Computing (SNIC) at the High Performance Computing Centre North (HPC2N).

\section{APPENDIX A: PAIR-CREATION RATE}

The probability rate of pair creation, $W_{ \pm}$, for a photon with energy $\omega$ and quantum nonlinearity parameter $\chi_{\gamma}=$ $e \sqrt{-(F k)^{2}} / m^{3}$ is conveniently expressed as

$$
W_{ \pm}=\frac{\alpha m^{2} \chi_{\gamma} T\left(\chi_{\gamma}\right)}{\omega}
$$

using the auxiliary function $[60,62]$

$$
T\left(\chi_{\gamma}\right)=\frac{1}{6 \sqrt{3} \pi \chi_{\gamma}} \int_{1}^{\infty} \frac{8 u+1}{u^{3 / 2} \sqrt{u-1}} K_{2 / 3}\left(\frac{8 u}{3 \chi_{\gamma}}\right) d u .
$$

(The integrand cannot be interpreted as a spectrum.) It has limits

$$
T\left(\chi_{\gamma}\right) \simeq\left\{\begin{array}{cl}
\frac{3 \sqrt{3}}{16 \sqrt{2}} \exp \left(-\frac{8}{3 \chi_{\gamma}}\right), & \chi_{\gamma} \ll 1 \\
\frac{0.37961}{\chi_{\gamma}^{1 / 3}}, & \chi_{\gamma} \gg 1,
\end{array}\right.
$$

and the following approximation from Erber [60] works well across the full range of $\chi_{\gamma}$ :

$$
T\left(\chi_{\gamma}\right) \simeq \frac{0.16}{\chi_{\gamma}} K_{1 / 3}^{2}\left(\frac{4}{3 \chi_{\gamma}}\right)
$$

\section{APPENDIX B: SIMULATIONS}

In Secs. III and IV A we compare our theoretical prediction with the results of single-particle Monte Carlo simulations, using the same code developed for and described in $[45,69]$. Each electron is followed along its trajectory through the laser pulse and QED events are sampled at every time step using the standard Monte Carlo approach [28]. The electron momentum is updated at every time step assuming that the external fields are constant and crossed, but the particle push is simplified to ballistic propagation at the speed of light. This requires $\gamma \gg a_{0}$ and restricts use of the code to collisions with externally imposed electromagnetic waves, but these approximations permit substantial speedup over conventional PIC codes.

In Sec. IV C we compare our theoretical predictions to the results of simulations performed with the $3 \mathrm{D}$ PIC code EPOCH [70]. The first-order QED processes of photon emission and pair creation are implemented via Monte Carlo sampling of rates calculated in the locally constant field approximation. The simulation domain is $-10<x / \mu \mathrm{m}<10$, $-10<y / \mu \mathrm{m}<10$, and $-10<z / \mu \mathrm{m}<30$, resolved with $(20,20,40)$ cells per micron in the $x, y$, and $z$ directions, respectively. The time step is set by the Courant-FriedrichsLewy condition, as there are sufficient cells to ensure that the probability of multiple QED events in a single time step is negligible [58]. The electron beam is initialized with a Gaussian charge density profile (FWHM $10 \mu \mathrm{m}$ ) centered at $x=\Delta, y=0$, and $z=24 \mu \mathrm{m}$ (where $\Delta$ is an offset between the beams) and represented with eight macroelectrons per cell for a total of $9.9 \times 10^{8}$ particles. The laser pulse is represented by a paraxial Gaussian beam (waist $2 \mu \mathrm{m}$, wavelength 0.8 $\mu \mathrm{m})$, is polarized along $x$, propagates towards positive $z$ with Gaussian temporal profile (intensity FWHM $30 \mathrm{fs}$ ), and is timed to reach focus when the electron beam center arrives at $z=0$. Final energy spectra for the collision parameters given in Sec. IV C are shown in Fig. 9.
[1] G. Breit and J. A. Wheeler, Phys. Rev. 46, 1087 (1934).

[2] O. J. Pike, F. Mackenroth, E. G. Hill, and S. J. Rose, Nat. Photon. 8, 434 (2014).

[3] X. Ribeyre, E. d'Humières, O. Jansen, S. Jequier, V. T. Tikhonchuk, and M. Lobet, Phys. Rev. E 93, 013201 (2016).

[4] C. Bula, K. T. McDonald, E. J. Prebys, C. Bamber, S. Boege, T. Kotseroglou, A. C. Melissinos, D. D. Meyerhofer, W. Ragg, D. L. Burke, R. C. Field, G. Horton-Smith, A. C. Odian, J. E. Spencer, D. Walz, S. C. Berridge, W. M. Bugg, K. Shmakov, and A. W. Weidemann, Phys. Rev. Lett. 76, 3116 (1996).

[5] D. L. Burke, R. C. Field, G. Horton-Smith, J. E. Spencer, D. Walz, S. C. Berridge, W. M. Bugg, K. Shmakov, A. W. Weidemann, C. Bula, K. T. McDonald, E. J. Prebys, C. Bamber, S. J. Boege, T. Koffas, T. Kotseroglou, A. C. Melissinos, D. D.
Meyerhofer, D. A. Reis, and W. Ragg, Phys. Rev. Lett. 79, 1626 (1997).

[6] C. Bamber, S. J. Boege, T. Koffas, T. Kotseroglou, A. C. Melissinos, D. D. Meyerhofer, D. A. Reis, W. Ragg, C. Bula, K. T. McDonald, E. J. Prebys, D. L. Burke, R. C. Field, G. Horton-Smith, J. E. Spencer, D. Walz, S. C. Berridge, W. M. Bugg, K. Shmakov, and A. W. Weidemann, Phys. Rev. D 60, 092004 (1999).

[7] The Extreme Light Infrastructure (ELI) project.

[8] The Vulcan 10 PW Project.

[9] The Exawatt Center for Extreme Light Studies (XCELS).

[10] G. Chériaux, F. Giambruno, A. Fréneaux, F. Leconte, L. P. Ramirez, P. Georges, F. Druon, D. N. Papadopoulos, A. Pellegrina, C. L. Blanc, I. Doyen, L. Legat, J. M. Boudenne, 
G. Mennerat, P. Audebert, G. Mourou, F. Mathieu, and J. P. Chambaret, AIP Conf. Proc. 1462, 78 (2012).

[11] H. Hu, C. Müller, and C. H. Keitel, Phys. Rev. Lett. 105, 080401 (2010).

[12] A. Ilderton, Phys. Rev. Lett. 106, 020404 (2011).

[13] B. King and H. Ruhl, Phys. Rev. D 88, 013005 (2013).

[14] P. Goldreich and W. H. Julian, Astrophys. J. 157, 869 (1969).

[15] T. Piran, Rev. Mod. Phys. 76, 1143 (2005).

[16] R. Ruffini, G. Vereshchagin, and S.-S. Xue, Phys. Rep. 487, 1 (2010).

[17] A. K. Harding and D. Lai, Rep. Prog. Phys. 69, 2631 (2006).

[18] G. A. Mourou, T. Tajima, and S. V. Bulanov, Rev. Mod. Phys. 78, 309 (2006).

[19] M. Marklund and P. K. Shukla, Rev. Mod. Phys. 78, 591 (2006).

[20] A. Di Piazza, C. Müller, K. Z. Hatsagortsyan, and C. H. Keitel, Rev. Mod. Phys. 84, 1177 (2012).

[21] H. Chen, S. C. Wilks, J. D. Bonlie, E. P. Liang, J. Myatt, D. F. Price, D. D. Meyerhofer, and P. Beiersdorfer, Phys. Rev. Lett. 102, 105001 (2009).

[22] H. Chen, F. Fiuza, A. Link, A. Hazi, M. Hill, D. Hoarty, S. James, S. Kerr, D. D. Meyerhofer, J. Myatt, J. Park, Y. Sentoku, and G. J. Williams, Phys. Rev. Lett. 114, 215001 (2015).

[23] E. Liang, T. Clarke, A. Henderson, W. Fu, W. Lo, D. Taylor, P. Chaguine, S. Zhou, Y. Hua, X. Cen, X. Wang, J. Kao, H. Hasson, G. Dyer, K. Serratto, N. Riley, M. Donovan, and T. Ditmire, Sci. Rep. 5, 13968 (2015).

[24] C. Gahn, G. D. Tsakiris, G. Pretzler, K. J. Witte, P. Thirolf, D. Habs, C. Delfin, and C.-G. Wahlström, Phys. Plasmas 9, 987 (2002).

[25] G. Sarri, W. Schumaker, A. Di Piazza, M. Vargas, B. Dromey, M. E. Dieckmann, V. Chvykov, A. Maksimchuk, V. Yanovsky, Z. H. He, B. X. Hou, J. A. Nees, A. G. R. Thomas, C. H. Keitel, M. Zepf, and K. Krushelnick, Phys. Rev. Lett. 110, 255002 (2013).

[26] G. Sarri, K. Poder, J. M. Cole, W. Schumaker, A. Di Piazza, B. Reville, T. Dzelzainis, D. Doria, L. A. Gizzi, G. Grittani, S. Kar, C. H. Keitel, K. Krushelnick, S. Kuschel, S. P. D. Mangles, Z. Najmudin, N. Shukla, L. O. Silva, D. Symes, A. G. R. Thomas, M. Vargas, J. Vieira, and M. Zepf, Nat. Commun. 6, 6747 (2015).

[27] A. R. Bell and J. G. Kirk, Phys. Rev. Lett. 101, 200403 (2008).

[28] R. Duclous, J. G. Kirk, and A. R. Bell, Plasma Phys. Control. Fusion 53, 015009 (2011).

[29] E. N. Nerush, I. Y. Kostyukov, A. M. Fedotov, N. B. Narozhny, N. V. Elkina, and H. Ruhl, Phys. Rev. Lett. 106, 035001 (2011).

[30] N. V. Elkina, A. M. Fedotov, I. Y. Kostyukov, M. V. Legkov, N. B. Narozhny, E. N. Nerush, and H. Ruhl, Phys. Rev. Spec. Top. Accel. Beams 14, 054401 (2011).

[31] M. Jirka, O. Klimo, S. V. Bulanov, T. Z. Esirkepov, E. Gelfer, S. S. Bulanov, S. Weber, and G. Korn, Phys. Rev. E 93, 023207 (2016).

[32] T. Grismayer, M. Vranic, J. L. Martins, R. A. Fonseca, and L. O. Silva, Phys. Rev. E 95, 023210 (2017).

[33] C. P. Ridgers, C. S. Brady, R. Duclous, J. G. Kirk, K. Bennett, T. D. Arber, A. P. L. Robinson, and A. R. Bell, Phys. Rev. Lett. 108, 165006 (2012).

[34] C. S. Brady, C. P. Ridgers, T. D. Arber, A. R. Bell, and J. G. Kirk, Phys. Rev. Lett. 109, 245006 (2012).

[35] X.-L. Zhu, T.-P. Yu, Z.-M. Sheng, Y. Yin, I. C. E. Turcu, and A. Pukhov, Nat. Commun. 7, 13686 (2016).
[36] S.-W. Bahk, P. Rousseau, T. A. Planchon, V. Chvykov, G. Kalintchenko, A. Maksimchuk, G. A. Mourou, and V. Yanovsky, Opt. Lett. 29, 2837 (2004).

[37] I. V. Sokolov, N. M. Naumova, J. A. Nees, and G. A. Mourou, Phys. Rev. Lett. 105, 195005 (2010).

[38] S. S. Bulanov, C. B. Schroeder, E. Esarey, and W. P. Leemans, Phys. Rev. A 87, 062110 (2013).

[39] X. Wang, R. Zgadzaj, N. Fazel, Z. Li, S. A. Yi, X. Zhang, W. Henderson, Y.-Y. Chang, R. Korzekwa, H.-E. Tsai, C.-H. Pai, H. Quevedo, G. Dyer, E. Gaul, M. Martinez, A. C. Bernstein, T. Borger, M. Spinks, M. Donovan, V. Khudik, G. Shvets, T. Ditmire, and M. C. Downer, Nat. Commun. 4, 1988 (2013).

[40] H. T. Kim, K. H. Pae, H. J. Cha, I. J. Kim, T. J. Yu, J. H. Sung, S. K. Lee, T. M. Jeong, and J. Lee, Phys. Rev. Lett. 111, 165002 (2013).

[41] W. P. Leemans, A. J. Gonsalves, H.-S. Mao, K. Nakamura, C. Benedetti, C. B. Schroeder, C. Tóth, J. Daniels, D. E. Mittelberger, S. S. Bulanov, J.-L. Vay, C. G. R. Geddes, and E. Esarey, Phys. Rev. Lett. 113, 245002 (2014).

[42] S. Chen, N. D. Powers, I. Ghebregziabher, C. M. Maharjan, C. Liu, G. Golovin, S. Banerjee, J. Zhang, N. Cunningham, A. Moorti, S. Clarke, S. Pozzi, and D. P. Umstadter, Phys. Rev. Lett. 110, 155003 (2013).

[43] G. Sarri, D. J. Corvan, W. Schumaker, J. M. Cole, A. Di Piazza, H. Ahmed, C. Harvey, C. H. Keitel, K. Krushelnick, S. P. D. Mangles, Z. Najmudin, D. Symes, A. G. R. Thomas, M. Yeung, Z. Zhao, and M. Zepf, Phys. Rev. Lett. 113, 224801 (2014).

[44] N. Neitz and A. Di Piazza, Phys. Rev. Lett. 111, 054802 (2013).

[45] T. G. Blackburn, C. P. Ridgers, J. G. Kirk, and A. R. Bell, Phys. Rev. Lett. 112, 015001 (2014).

[46] V. Dinu, C. Harvey, A. Ilderton, M. Marklund, and G. Torgrimsson, Phys. Rev. Lett. 116, 044801 (2016).

[47] M. Vranic, T. Grismayer, R. A. Fonseca, and L. O. Silva, New J. Phys. 18, 073035 (2016).

[48] T. Heinzl, A. Ilderton, and M. Marklund, Phys. Lett. B 692, 250 (2010).

[49] B. King, N. Elkina, and H. Ruhl, Phys. Rev. A 87, 042117 (2013).

[50] K. Krajewska, C. Müller, and J. Z. Kamiński, Phys. Rev. A 87, 062107 (2013).

[51] V. Dinu, T. Heinzl, A. Ilderton, M. Marklund, and G. Torgrimsson, Phys. Rev. D 89, 125003 (2014).

[52] S. Meuren, K. Z. Hatsagortsyan, C. H. Keitel, and A. Di Piazza, Phys. Rev. D 91, 013009 (2015).

[53] S. Meuren, C. H. Keitel, and A. Di Piazza, Phys. Rev. D 93, 085028 (2016).

[54] A. Di Piazza, Phys. Rev. Lett. 117, 213201 (2016).

[55] A. Di Piazza, Phys. Rev. A 95, 032121 (2017).

[56] A. A. Mironov, N. B. Narozhny, and A. M. Fedotov, Phys. Lett. A 378, 3254 (2014).

[57] M. Lobet, X. Davoine, E. d'Humières, and L. Gremillet, Phys. Rev. Accel. Beams 20, 043401 (2017).

[58] C. P. Ridgers, J. G. Kirk, R. Duclous, T. G. Blackburn, C. S. Brady, K. Bennett, T. D. Arber, and A. R. Bell, J. Comput. Phys. 260, 273 (2014).

[59] A. Gonoskov, S. Bastrakov, E. Efimenko, A. Ilderton, M. Marklund, I. Meyerov, A. Muraviev, A. Sergeev, I. Surmin, and E. Wallin, Phys. Rev. E 92, 023305 (2015).

[60] T. Erber, Rev. Mod. Phys. 38, 626 (1966).

[61] V. I. Ritus, J. Sov. Laser Res. 6, 497 (1985). 
[62] V. N. Baier, V. M. Katkov, and V. M. Strakhovenko, Electromagnetic Processes at High Energies in Oriented Single Crystals (World Scientific, Singapore, 1998).

[63] W. Heisenberg and H. Euler, Z. Phys. 98, 714 (1936).

[64] A. A. Sokolov and I. M. Ternov, in Synchrotron Radiation, edited by E. Schmutzer (Pergamon, New York, 1968).

[65] C. S. Shen and D. White, Phys. Rev. Lett. 28, 455 (1972).

[66] C. N. Harvey, A. Gonoskov, A. Ilderton, and M. Marklund, Phys. Rev. Lett. 118, 105004 (2017).
[67] J. Schwinger, Proc. Natl. Acad. Sci. USA 40, 132 (1954).

[68] J. Lindhard, Phys. Rev. A 43, 6032 (1991).

[69] T. G. Blackburn, Plasma Phys. Control. Fusion 57, 075012 (2015).

[70] T. D. Arber, K. Bennett, C. S. Brady, A. Lawrence-Douglas, M. G. Ramsay, N. J. Sircombe, P. Gillies, R. G. Evans, H. Schmitz, A. R. Bell, and C. P. Ridgers, Plasma Phys. Control. Fusion 57, 113001 (2015). 